\title{
A diabatically-generated potential vorticity structure near the extratropical tropopause in three simulated extratropical cyclones
}

Article

Published Version

Chagnon, J. M. and Gray, S. L. (2015) A diabaticallygenerated potential vorticity structure near the extratropical tropopause in three simulated extratropical cyclones. Monthly Weather Review, 143 (6). pp. 2337-2347. ISSN 0027-0644 doi: https://doi.org/10.1175/MWR-D-14-00092.1 Available at https://centaur.reading.ac.uk/39241/

It is advisable to refer to the publisher's version if you intend to cite from the work. See Guidance on citing.

Published version at: http://dx.doi.org/10.1175/MWR-D-14-00092.1

To link to this article DOI: http://dx.doi.org/10.1175/MWR-D-14-00092.1

Publisher: American Meteorological Society

All outputs in CentAUR are protected by Intellectual Property Rights law, including copyright law. Copyright and IPR is retained by the creators or other copyright holders. Terms and conditions for use of this material are defined in the End User Agreement.

www.reading.ac.uk/centaur 
Central Archive at the University of Reading

Reading's research outputs online 


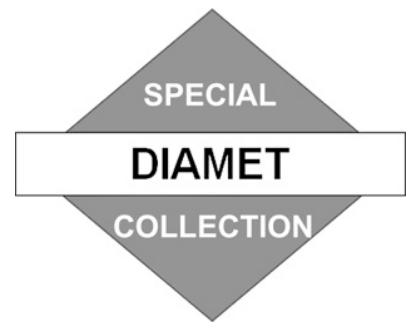

\title{
A Diabatically Generated Potential Vorticity Structure near the Extratropical Tropopause in Three Simulated Extratropical Cyclones
}

\author{
JEFFREY M. CHAGNON \\ Department of Earth, Ocean, and Atmospheric Science, Florida State University, Tallahassee, Florida \\ SUZANNE L. GRAY \\ University of Reading, Reading, United Kingdom
}

(Manuscript received 11 March 2014, in final form 7 November 2014)

\begin{abstract}
The structure of near-tropopause potential vorticity (PV) acts as a primary control on the evolution of extratropical cyclones. Diabatic processes such as the latent heating found in ascending moist warm conveyor belts modify PV. A dipole in diabatically generated PV (hereafter diabatic PV) straddling the extratropical tropopause, with the positive pole above the negative pole, was diagnosed in a recently published analysis of a simulated extratropical cyclone. This PV dipole has the potential to significantly modify the propagation of Rossby waves and the growth of baroclinically unstable waves. This previous analysis was based on a single case study simulated with $12-\mathrm{km}$ horizontal grid spacing and parameterized convection. Here the dipole is investigated in three additional cold-season extratropical cyclones simulated in both convectionparameterizing and convection-permitting model configurations. A diabatic PV dipole across the extratropical tropopause is diagnosed in all three cases. The amplitude of the dipole saturates approximately $36 \mathrm{~h}$ from the time diabatic PV is accumulated. The node elevation of the dipole varies between 2 and 4 PVU (1 PVU $\left.=10^{6} \mathrm{~K} \mathrm{~kg}^{-1} \mathrm{~m}^{2} \mathrm{~s}^{-1}\right)$ in the three cases, and the amplitude of the system-averaged dipole varies between 0.2 and 0.4 PVU. The amplitude of the negative pole is similar in the convection-parameterizing and convection-permitting simulations. The positive pole, which is generated by longwave radiative cooling, is weak in the convection-permitting simulations due to the small domain size, which limits the accumulation of diabatic tendencies within the interior of the domain. The possible correspondence between the diabatic PV dipole and the extratropical tropopause inversion layer is discussed.
\end{abstract}

\section{Introduction}

Heating and cooling due to diabatic processes result in changes to stratification and generation of flow anomalies through dynamic adjustment toward balance. Such changes are manifest as modifications to the potential vorticity (PV). The consequences of diabatic modification of PV (hereafter diabatic PV) in extratropical cyclones have been investigated in numerous contexts, including rapid cyclogenesis (e.g., Kuo et al. 1991; Stoelinga 1996;

Corresponding author address: Jeffrey M. Chagnon, Department of Earth, Ocean, and Atmospheric Science, Florida State University, 1017 Academic Way, Tallahassee, FL 32306.

E-mail: jchagnon@fsu.edu
Wernli and Davies 1997), diabatic Rossby waves (e.g., Parker and Thorpe 1995; Moore and Montgomery 2004; Moore et al. 2013), and forecast error growth (e.g., Brennan et al. 2008; Davies and Didone 2013).

In the absence of frictional and diabatic processes, $\mathrm{PV}$ is materially conserved. Therefore, PV provides a convenient means to characterize the large-scale structure of the atmosphere and its evolution in mid- and high latitudes where the planetary vorticity is nonnegligible. Climatologically, PV increases with height on average, and the gradient of PV increases abruptly at about the height of the thermal tropopause with the increased stratification above acting as a transport barrier. As such, the tropopause elevation is often approximated as the elevation of a surface of constant PV [typically in the 
range 1.5-4 PVU $\left.\left(1 \mathrm{PVU}=10^{6} \mathrm{~K} \mathrm{~kg}^{-1} \mathrm{~m}^{2} \mathrm{~s}^{-1}\right)\right]$. The isentropic gradient of $\mathrm{PV}$ at the level of the tropopause provides a waveguide for propagating Rossby waves. Dry baroclinic instability involves the interaction of these waves near the tropopause with counterpropagating waves in the lower troposphere (Heifetz et al. 2004). In moist baroclinic instability, diabatic heating introduces additional PV components (e.g., at the top and bottom of a heated layer), which may interact with the dry Rossby wave components via the induced velocities. De Vries et al. (2010) showed that this interaction results in a largeramplitude upper-level PV wave and a larger growth rate of the baroclinic wave. Given the uncertain nature of the parameterized processes that supply diabatic heating and cooling in numerical weather prediction models, it is of critical importance to determine the mechanisms by which these diabatic uncertainties influence the PV and development of extratropical cyclones.

Chagnon et al. (2013, hereafter CGM13) diagnosed a dipole pattern of diabatic PV straddling the tropopause in global and mesoscale model simulations of a North Atlantic cyclone using a PV tracer method. CGM13 demonstrated that

(i) the diabatic PV was near zero on average where the background PV was approximately $2 \mathrm{PVU}$, and

(ii) the diabatic PV was positive above and negative beneath the 2-PVU surface.

These two results, respectively, imply that

(i) diabatic PV did not directly lead to a change in tropopause elevation, and

(ii) the diabatic PV was in phase with the PV of the environment.

These conclusions in turn suggest that diabatic processes may influence moist baroclinic instability through the coupling of a diabatic PV wave to the Rossby wave near the tropopause, as described in De Vries et al. (2010). CGM13 (their section 5.1) describe this coupling mechanism and its relevance to the PV dipole in more detail. The analysis of CGM13 was based on a single case study simulated with the Met Office Unified Model (MetUM) run at a resolution that necessitated the use of a convection parameterization scheme. Done et al. (2006) demonstrated that the structure of PV in simulations of mesoscale convective systems is sensitive to whether convection is explicit or parameterized suggesting that the diabatic PV dipole found in CGM13 might be similarly sensitive.

The aim of this study is to examine the diabatic PV near the tropopause in three additional cases simulated at both convection-parameterizing and convectionpermitting resolution. The questions addressed by the analysis of additional cases are the following: (i) Is the dipole in PV straddling the tropopause a feature common to other extratropical cyclones?

(ii) Do the characteristics of the dipole depend on the horizontal grid spacing and the related changes in convection parameterization?

(iii) What are the time and spatial scales that characterize the production and maintenance of the dipole?

The structure of the paper is as follows. Section 2 describes the methods of analysis, including the configurations of the model runs and the PV tracer method. The basic characteristics of the three cases are also described in section 2. Section 3 examines the structure of the dipole in diabatic PV across the tropopause in all three cases simulated at convection-permitting and convectionparameterizing resolution. Section 4 provides a summary of results and a discussion of their implications.

\section{Method}

The analysis of diabatic PV across the extratropical tropopause is performed in numerical simulations of three extratropical cyclones in the North Atlantic. Simulations of each case were performed using the MetUM, version 7.3, the details of which are provided below along with a description of the PV tracer diagnostic. A more detailed description of the PV tracer method is provided in CGM13.

\section{a. MetUM and PV tracers}

The MetUM is a numerical weather prediction system utilized for weather and climate forecasting by the Met Office. It consists of a dynamical core that approximates solutions to the nonhydrostatic and fully compressible equations of motion on a sphere using a semi-implicit semiLagrangian technique, along with a suite of parameterization schemes to represent subgridscale processes. The model uses an Arakawa $\mathrm{C}$ grid in the horizontal with Charney-Phillips staggering in the vertical. The MetUM utilizes a height-based terrain-following vertical coordinate. Davies et al. (2005) provide a comprehensive summary of the model's design. The parameterization schemes include the mass-flux convection scheme of Gregory and Rowntree (1990), the MOSES-II boundary layer scheme (Lock et al. 2000), the Edwards and Slingo (1996) radiation scheme, and the cloud microphysics scheme of Wilson and Ballard (1999). The schemes used in this study are configured in the same way as the operational MetUM, version 7.3, which was used by the Met Office.

Table 1 summarizes the design of the simulations. Each of the three cases investigated in this study is simulated with the MetUM in three different configurations having horizontal grid spacings of 12,4 , and $1 \mathrm{~km}$. These simulations are hereafter referred to as the 12-, 4-, and $1-\mathrm{km}$ runs, respectively. The $12-$ and $4-\mathrm{km}$ runs are 
TABLE 1. Summary of MetUM experiments.

\begin{tabular}{ccccl}
\hline \hline Run name & Vertical levels & Convection scheme & Dynamics domain & \multicolumn{1}{c}{ Tracer domain(s) } \\
\hline $12 \mathrm{~km}$ & 38 & On & Large & Large, medium, small \\
$4 \mathrm{~km}$ & 38 & Modified & Medium & Medium, small \\
$1 \mathrm{~km}$ & 76 & Off & Small & Small \\
\hline
\end{tabular}

performed for $48 \mathrm{~h}$, and the 1-km runs are performed for $24 \mathrm{~h}$. The 12- and 4-km runs are performed with the convection scheme enabled; the $1-\mathrm{km}$ run is performed with the convection scheme turned off. In the 4-km runs, the convection scheme is modified such that the CAPE closure time scale is specified as an increasing function of CAPE, which ensures that the scheme is most efficient at removing instability near the grid scale (Roberts 2003). The 12-km runs utilize 38 nonconstantly spaced vertical levels between the lower boundary and the model top located at $39-\mathrm{km}$ elevation. The $4-$ and $1-\mathrm{km}$ runs utilize 76 nonconstantly spaced levels over the same depth. The designs of the grid domains for each run are discussed in section $2 b$. The high-resolution domains used here are not the same as those that have been used operationally by the Met Office; they have been tailored to encompass the downstream side of the upper-level trough where the broadest regions of cloud, precipitation, and latent heating are located. Initial conditions for the $12-\mathrm{km}$ runs are provided by an operational analysis from the Met Office at the same resolution, and lateral boundary conditions are provided by an operational run from the global MetUM with $\sim 40-\mathrm{km}$ resolution and 38 levels. The 4 - and $1-\mathrm{km}$ runs receive lateral boundary conditions from the 12- and 4-km runs, respectively, via one-way nesting.

Diagnosis of accumulated PV sources is performed using a PV tracer diagnostic, as described in detail in CGM13. The method is similar to that used in Stoelinga (1996). While CGM13 partitioned the diabatic PV in the model among contributions from various parameterized processes, the analysis in this investigation is restricted to only two PV fields. The first is the total PV, calculated from the full wind, density, and potential temperature fields at each time step. The second is an advection-only PV tracer, referred to as the conservative PV. This field is calculated using a passive tracer in the MetUM that is initialized with the full PV field at the beginning of the model run and is subsequently advected without additional sources by the model's advection scheme. The conservative PV tracer represents the structure of the PV that would result if no diabatic processes were directly modifying the PV; however, the conservative tracer is still influenced indirectly by diabatic processes that modify the wind field used to advect the tracer. The difference between the full PV and conservative PV tracer represents the net accumulated effect of diabatic tendencies on the
PV. This difference field will be referred to as the "net diabatic PV." The PV tracers are subject to implicit diffusion associated with the model's semi-Lagrangian advection scheme. As reported in CGM13, the numerical diffusion introduces amplitude errors of approximately $25 \%$ after 5 days of integration time. Errors of similar size can be attributed to inconsistencies in the manner by which conserved PV advection increments are calculated (L. Saffin 2014, personal communication). The conserved (adiabatic) advection increments can be calculated by either advecting a passive PV tracer (as is done in this study) or by computing the adiabatically advected increments in velocity, potential temperature, and density separately in order to construct an advection-only PV increment. For the 48-h simulations presented in this study, the tracer method is reliable despite these uncertainties because of numerical diffusion and tracer advection.

For the lateral boundary conditions, the conservative tracer is set to the value of the full PV and the diabatic $\mathrm{PV}$ is set to zero, thus restricting the accumulation of net diabatic PV to the interior of the tracer domain. To enable a comparison between runs performed at different resolutions, three tracer domains are defined: large, medium, and small corresponding to the domains of the $12-, 4-$, and $1-\mathrm{km}$ runs, respectively. The $12-\mathrm{km}$ runs are each performed three times with tracer accumulated in the large, medium, and small tracer domains (i.e., for the medium and small tracer domain runs the diabatic PV is set to zero on the boundary and only accumulated within the required subdomain of the domain over which the model is integrated). The 4-km runs are each performed twice with tracer accumulated in the medium and small tracer domains. The 1-km runs are performed only once with the tracer accumulated in the small tracer domain.

\section{b. Overview of case studies}

The three cases chosen for analysis occurred on 23 September 2011 (case I), 29 November 2011 (case II), and 24 November 2009 (case III). The three cases were selected based on the presence of an amplifying upper-level wave pattern and a well-defined warm conveyor belt (WCB) cloud band. Each case corresponded to an intensive observing periods (IOPs) 3 and 5 of the Diabatic Influences on Mesoscale Structures in Extratropical Storms (DIAMET) project field campaign and IOP3 of the THORPEX North Atlantic Waveguide and Downstream 

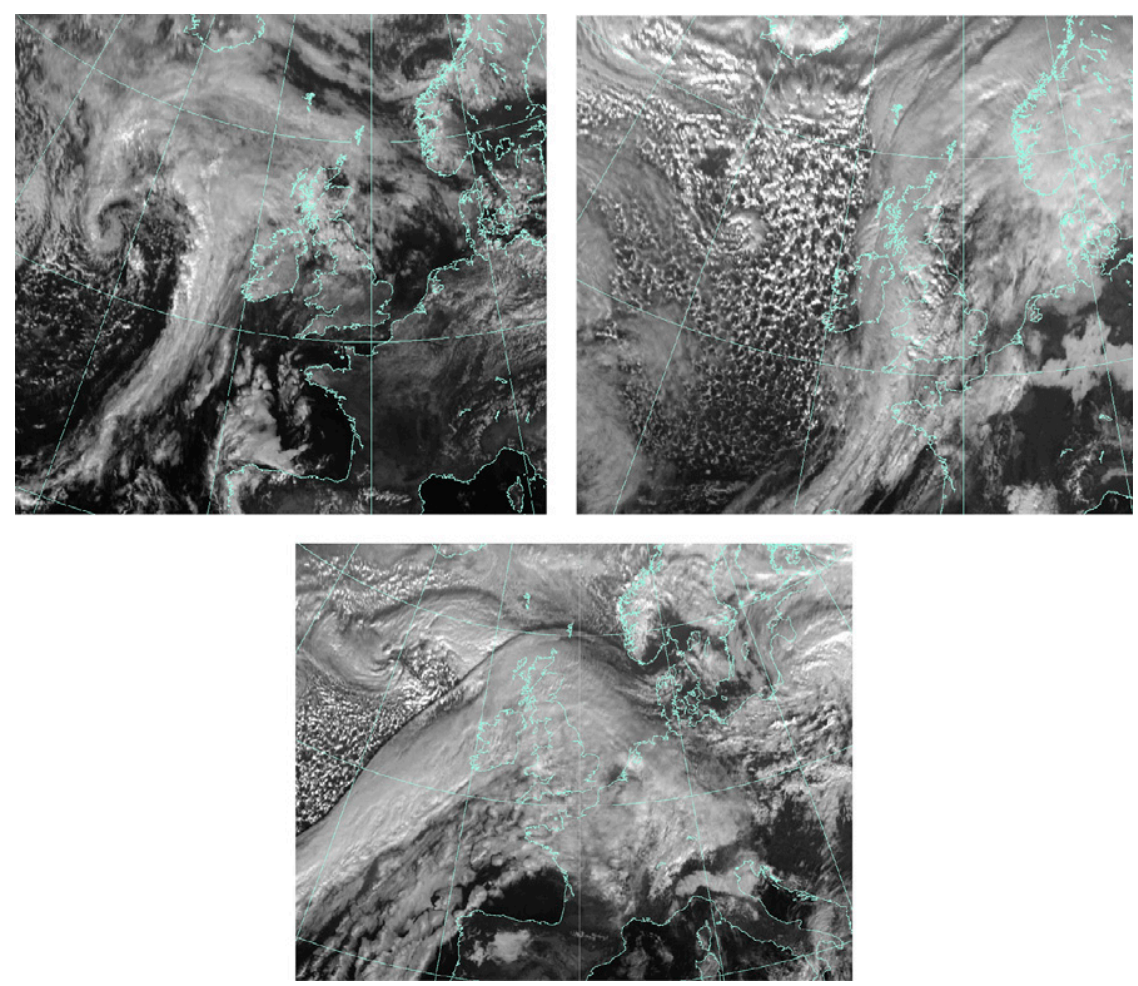

FIG. 1. Meteosat visible channel satellite images over the North Atlantic and western Europe, valid at (a) 1200 UTC 23 Sep 2011 (case I), (b) 1200 UTC 29 Nov 2011 (case II), and (c) 1100 UTC 24 Nov 2009 (case III). Images provided courtesy of EUMETSAT.

Experiment (T-NAWDEX) pilot field campaign, respectively; the authors were investigators in both of these projects. Each of these cases has been the subject of investigation beyond that which is presented in this paper: for T-NAWDEX IOP3 see Martinez-Alvarado et al. (2014) and for IOP 5 see CGM13. The initialization times for the $12-\mathrm{km}$ runs in cases I, II, and III are 0000 UTC 23 September 2011, 1200 UTC 28 November 2011, and 1200 UTC 23 November 2009, respectively. Figure 1 shows visible satellite images of the primary cyclone in each of the three cases. A broad north-south- or southwest-northeastoriented cloud band is evident in each case, indicating the presence of a WCB (Harrold 1973) that formed along the cold front and on the downstream side of the upper-level trough. The WCB is an ascending airstream located along and above the backward-sloping cold frontal interface in which large quantities of heat and moisture are transported from the boundary layer poleward and into the upper troposphere. These features are typical of extratropical cyclones in the North Atlantic.

Figure 2 presents the full $\mathrm{PV}$ in each of the three cases after $24 \mathrm{~h}$ of integration time in the $12-\mathrm{km}$ runs (with tracer integrated over the large domain) on a model level that intersects the tropopause in the midlatitudes at $9.7-\mathrm{km}$ elevation. Each case is characterized by a large amplitude upper-level wave at this analysis time and the three cases are qualitatively similar with respect to structure and evolution of the large-scale trough and ridge pattern in the upper troposphere. The 12-km domains are identical for cases I and II, but this domain is slightly larger for case III [the larger domain was used to facilitate an intermodel comparison of a warm conveyor belt as reported in MartinezAlvarado et al. (2014)]. The subdomains are positioned along the downstream edge of the upper-level trougha region that captures the WCB where large amounts of precipitation, diabatic heating, and diabatic PV tendencies are found. Computational constraints limited the size of the domains used for the 4- and 1-km runs. For case I a larger 4-km domain was utilized. The presentation of results for the higher-resolution runs will focus on case I; however, the 4- and 1-km runs for cases II and III are qualitatively similar.

\section{Comparison of the PV dipole across the tropopause}

In this section, the net diabatic PV near the tropopause is compared in each of the three cases and in each of the three model configurations. 
a)

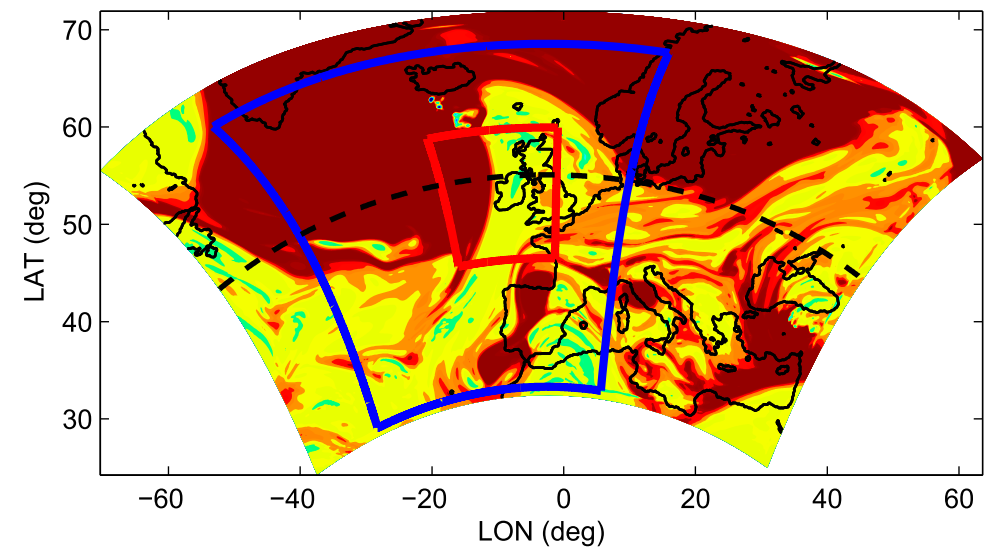

b)

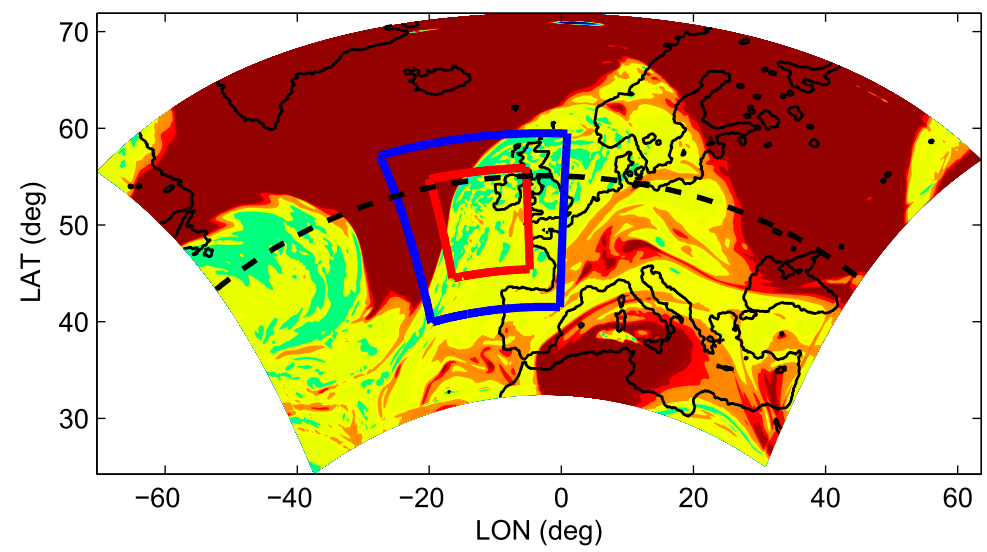

c)

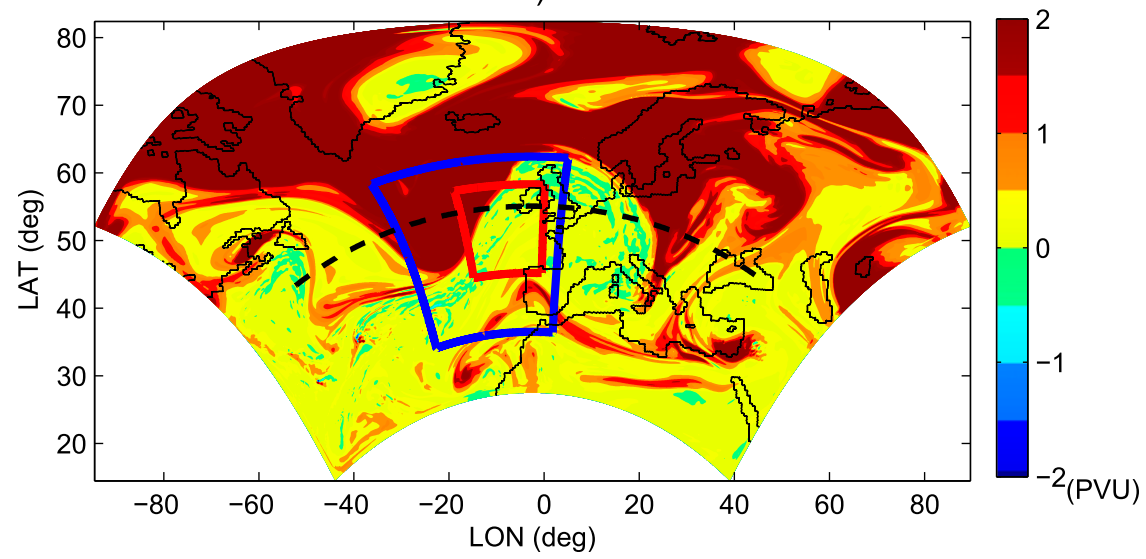

FIG. 2. Potential vorticity in the $12-\mathrm{km}$ runs on a model level corresponding to $9.68 \mathrm{~km}$ above ground level, valid $24 \mathrm{~h}$ into each simulation, for (a) case I, (b) case II, and (c) case III. The locations of the nested grid domains used in each case are indicated by the blue boxes for the $4-\mathrm{km}$ runs, and by the red boxes for the $1-\mathrm{km}$ runs. The dashed lines indicate the locations of the vertical cross sections shown in Fig. 3.

The net diabatic PV after $36 \mathrm{~h}$ of integration time in the $12-\mathrm{km}$ runs (with large tracer domain) is plotted in Fig. 3 for each of the three cases. The vertical sections chosen for presentation cross the core of the upper-level trough and ridge. In each case, the large-scale structure of the tropopause in the section is similar (i.e., the tropopause is lowered in the core of the trough and extends upward to $10-12-\mathrm{km}$ elevation in the ridge). The pattern 
a)

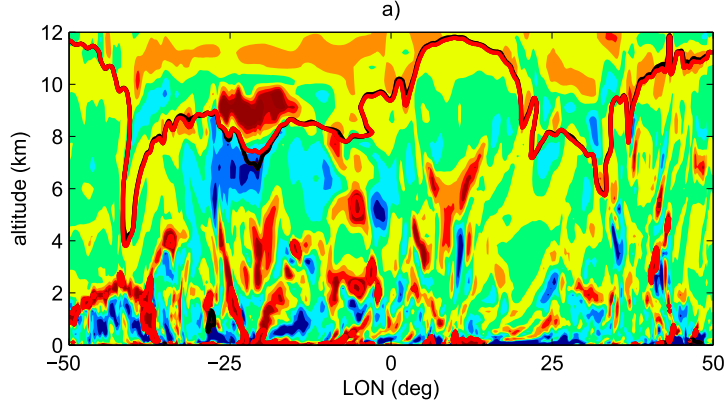

b)

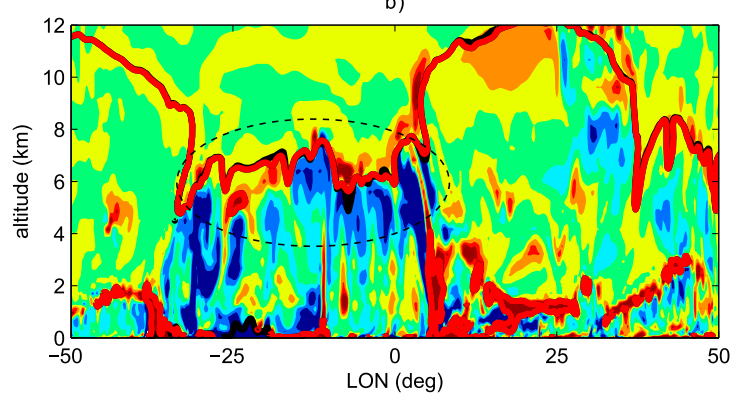

c)

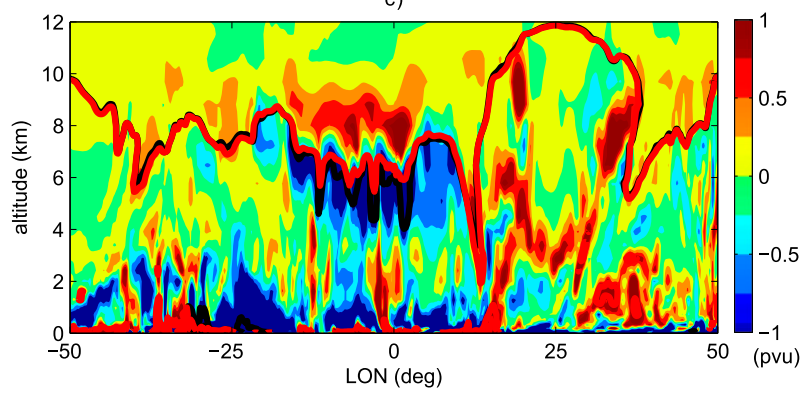

FIG. 3. Vertical cross sections of net accumulated diabatic PV (colored filled contours) valid $36 \mathrm{~h}$ into each simulation in (a) case I, (b) case II, and (c) case III. The 2-PVU contour of the full PV field is depicted by the red line, and the 2-PVU contour of the conservative PV tracer is depicted by the black line. The locations of the cross sections are indicated in Fig. 2. The dashed circle in (b) depicts the location of the dipole referred to in the text.

of net diabatic PV relative to the tropopause is also very similar in each case, and to that in CGM13; a broad region of negative diabatic PV is present beneath the tropopause (particularly in the trough, e.g., between $-20^{\circ}$ and $0^{\circ}$ longitude; see the dashed circle in Fig. $3 \mathrm{~b}$ for location of this dipole) and a shallower but broad region of positive diabatic $\mathrm{PV}$ is present above the tropopause. In CGM13, it was demonstrated that this negative diabatic PV resulted from contributions from the largescale cloud, convection, and boundary layer parameterization schemes whereas this positive diabatic PV was primarily contributed by the model's longwave radiation scheme. Commensurate with its mechanism of generation, the negative diabatic PV beneath the tropopause is noisier and is characterized by features having a smaller

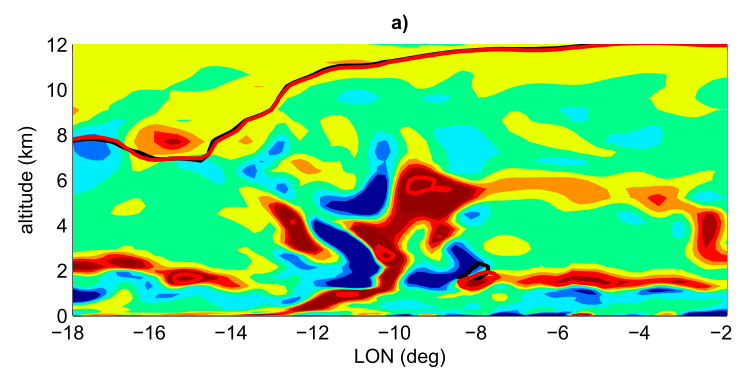

b)

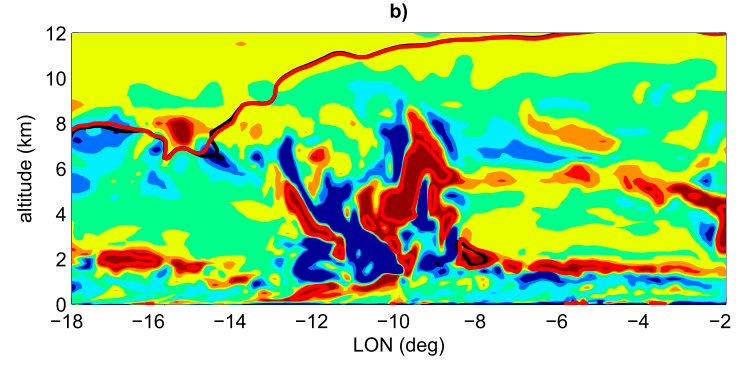

c)

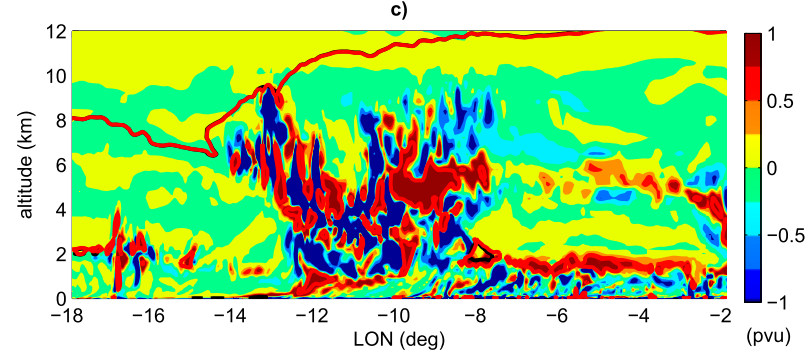

FIG. 4. As in Fig. 3, but for (a) the 12-, (b) the 4-, and (c) the 1-km runs $15 \mathrm{~h}$ into the simulation of case $\mathrm{I}$. Tracer is integrated over the large, medium, and small tracer domains, respectively. To enable comparison, the range of longitudes is restricted to that of the small tracer domain.

length scale than the smoother pattern of positive diabatic PV above the tropopause. Although these qualitative features are common to all three cases, case II is different from the other two cases in two respects; the elevation of the tropopause in the ridge is lowest in case II, and the depth of the PV dipole is shallowest in case II. The shallower PV dipole in case II is also accompanied by a sharper vertical gradient in humidity across the tropopause in comparison to cases I and III and a sharp increase in static stability above the tropopause (not shown). These features suggest that a shallow tropopause inversion layer was present in case II. The correspondence between the PV dipole and the tropopause inversion layer is discussed in section 4 .

A comparison of the diabatic PV in the 12-, 4-, and 1-km runs is presented in Fig. 4 for case I after $15 \mathrm{~h}$ (a shorter lead time is shown here than in Fig. 3 due to the shorter run time of the $1-\mathrm{km}$ runs). To enable a fair comparison, the fields are shown in a vertical section across the 1-km domain although the tracer is integrated 
a)

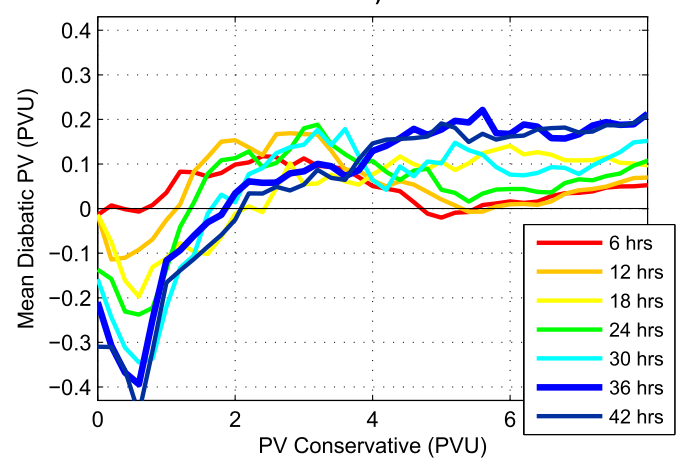

c)

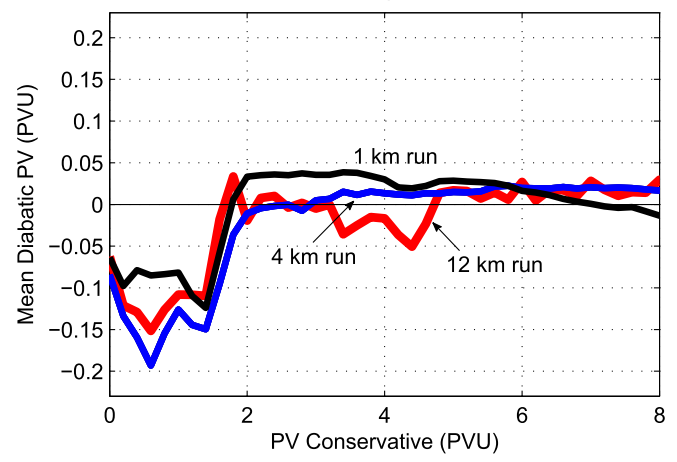

b)

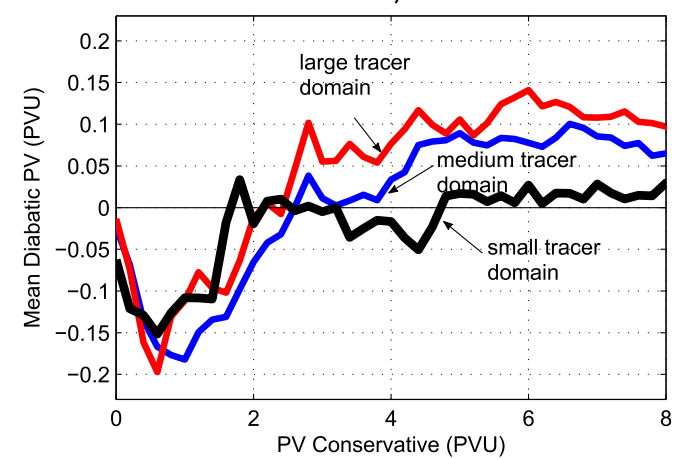

d)

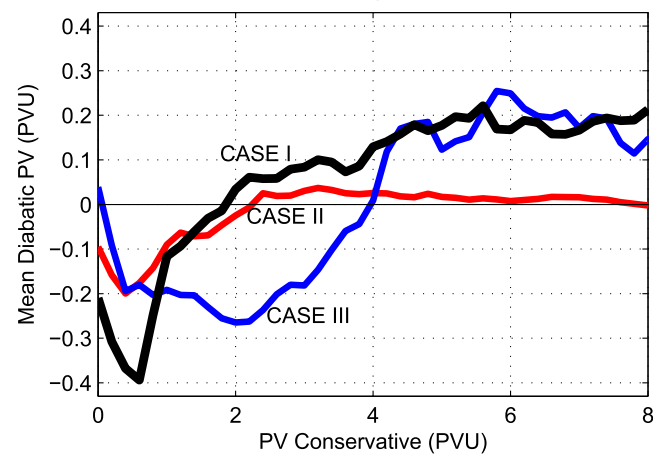

FIG. 5. Profiles of the average near-tropopause diabatic PV as a function of the conservative PV. The sensitivity of the dipole structure to four factors is demonstrated: (a) time evolution from 6 to $42 \mathrm{~h}$ in the $12-\mathrm{km}$ run of case I; (b) size of the tracer integration domain in the 12-km run of case I; (c) model grid resolution in case I; and (d) case for the $12-\mathrm{km}$ runs. The profiles in (b),(c), and (d) are valid after 36,18, and $36 \mathrm{~h}$ of tracer integration time, respectively.

over the full domains at each resolution (i.e., the large, medium, and small tracer domains for the 12-, 4- and 1-km runs, respectively). The qualitative agreement in net diabatic PV production between the three resolutions is good, the most notable difference being that smaller-scale structures are generated in the $1-\mathrm{km}$ run (cf. Figs. $4 \mathrm{a}, \mathrm{b}$ to Fig. 4c). However, there exist quantitative differences in the structure and amplitude of the negative portion of the dipole (see Fig. $5 \mathrm{c}$ and the related discussion below). These differences are likely to reflect differences in the representation of parameterized processes that contribute to latent heating in the three model configurations. In the 12- and 4-km runs, some diabatic PV structures are evident in the boundary layer near the western lateral boundary that are not present in the 1-km run; this lowertropospheric dipole is advected into the cross section in the coarser resolution runs with their larger domains. The large amplitude negative diabatic PV beneath the trough is simulated with a consistent structure and amplitude across all three resolutions.

To quantify the comparison of diabatic PV, Fig. 5 presents the mean profiles of net diabatic PV as a function of the conservative PV. The conservative PV serves as a vertical coordinate in which the tropopause is located between 2 and 4 PVU; values less than 2 PVU in the conservative PV correspond to elevations located in the troposphere. The profiles in Fig. 5 were computed by first sorting the conservative PV into bins of width 0.25 PVU (starting at 0 PVU), and then computing the area-averaged net diabatic PV for all points within each bin. The computation was restricted to points lying within $2.5 \mathrm{~km}$ in height from the 2-PVU surface. The depth of $2.5 \mathrm{~km}$ was chosen to ensure that the average includes the dipole across the tropopause, but excludes any unrelated PV structures in the lower troposphere (e.g., in the boundary layer or along frontal interfaces). The sensitivity of the net diabatic PV profile was tested to four different factors, each corresponding to one of the panels in Fig. 5: time (Fig. 5a), size of the tracer integration domain (i.e., spatial scale) (Fig. 5b), grid resolution (Fig. 5c), and case (Fig. 5d). To simplify the presentation, Figs. 5a-c are restricted to case I, but the conclusions drawn are valid in all three cases (not shown).

The evolution of the diabatic PV dipole across the tropopause is demonstrated in Fig. $5 \mathrm{a}$ for the $12-\mathrm{km}$ run of case I with large tracer domain. The amplitude of the dipole (defined as the larger of the magnitudes of the positive and negative poles) increases with time as 
the diabatic PV material accumulates. The stratospheric portion of the dipole increases in amplitude more slowly than the tropospheric portion, reflecting the relative importance of slower-acting processes (i.e., radiation) in the generation of the positive portion of the dipole above the tropopause. The amplitude of the dipole asymptotes toward approximately $0.3 \mathrm{PVU}$ on average in the first $36 \mathrm{~h}$ of integration. After this time the amplitude saturates, implying that additional processes (e.g., vertical advection and diffusion) are compensating the diabatic PV tendencies to establish a near steady-state dipole structure.

The dependence of the dipole on spatial scale is demonstrated in Fig. 5b. Each curve is calculated from a 12-km run of case I but with tracers integrated over the large, medium, and small tracer domains, respectively (see Fig. 2 for the domain specification). The primary difference between the three curves is in the lower stratosphere where the positive pole has a larger magnitude for tracers integrated over larger domains. The differences between the negative poles in the troposphere are comparatively small. The processes responsible for the generation of the negative pole are rapid and local, whereas those responsible for the positive pole are slower and act across a larger scale. CGM13 demonstrated a similar result in which longwave radiation generated positive net diabatic PV above the tropopause across both the upper-level ridge and trough. Although the cooling rates associated with longwave radiation are weaker than the heating rates associated with latent heating in the troposphere, the steady and continuous action of radiation across the upper-level wave renders its contribution to the net diabatic PV to be large at long lead times (greater than a day).

The effect of grid resolution on the structure of the dipole is demonstrated in Fig. 5c. The curves present the dipole generated in the 12-, 4-, and 1-km runs of case I all using the small tracer domain. Directly beneath the tropopause (i.e., between 1.5 and 2 PVU) the amplitude of the negative pole varies by approximately $25 \%$ between the run, although there is less agreement at lower $\mathrm{PV}$ values in the troposphere. Because each of the curves in Fig. $5 \mathrm{c}$ is calculated over the small tracer domain, none of them demonstrate a large positive signal above the tropopause. As discussed above, the positive pole is contributed primarily by longwave radiative cooling acting slowly but continually across the full extent of the upper-level wave, and the small tracer domain is not large enough to enclose its production.

The variation in dipole structure across the three cases is demonstrated in Fig. 5d using the 12-km runs with large tracer domains. A dipole in net diabatic PV straddling the tropopause is evident in all cases. However, the amplitude of the dipole and the PV level at which the net diabatic PV is zero varies between the cases. The positive pole is far weaker in case II than in the other two cases. As mentioned above, case II is also characterized by a tropopause that is lowest in elevation and a PV dipole that is shallower than in the other two cases (see Fig. 3). In case III, the node of the dipole is notably higher (4 PVU) than in cases I and II (2 PVU).

\section{Conclusions and discussion}

CGM13 presented an analysis of diabatically generated PV sources in a simulated extratropical cyclone. Their primary result was the identification of a dipole in accumulated diabatic PV straddling the tropopause. The dipole structure implied an increase in the vertical gradient of PV across the tropopause, which has important implications for the propagation of Rossby waves in the upper troposphere. The purpose of the present study was to investigate the dipole structure in three additional cases each simulated with three model resolutions ranging from convection parameterizing to convection permitting.

The dipole in diabatic PV across the tropopause has been shown to emerge in all of the 12- and 4-km simulations when the diabatic PV is averaged across the upper-level synoptic-scale wave in the large and medium tracer domains. We, therefore, conclude that the dipole is a common feature across the extratropical tropopause in an extratropical cyclone in the North Atlantic. The dipole is likely to be a feature common to extratropical cyclones in other regions, but its amplitude and structure may vary geographically due to variations in the mean humidity gradient across the tropopause and the availability of moisture in the troposphere. In the 1-km simulations and in the simulations performed in the small tracer domain, only the negative pole was clearly evident. It is hypothesized that the small domain size did not permit accumulation of positive tendencies due to longwave radiative cooling, which acted more slowly and on a larger scale than the negative tendencies associated with latent heating in the troposphere. The small domain size was nevertheless large enough to encompass PV modification by latent heating in the troposphere; the mean amplitude of the resulting negative diabatic PV varied by approximately $25 \%$ between the 12-, 4-, and 1-km simulations directly beneath the tropopause. The amplitude and the node of the dipole were shown to vary between the cases: the amplitude varied between 0.2 and $0.4 \mathrm{PVU}$ and the background PV value of the node varied between 2 and 4 PVU. This range is consistent with the range of dynamical tropopause PV values of 1.5-5 PVU estimated by Kunz et al. 
(2011). Furthermore, Hoerling et al. (1991) determined that the thermal tropopause is on average located near 3.5 PVU in the extratropics. We present two hypothesized mechanisms causing the node of the dipole in diabatic PV to be close to the tropopause height: (i) the vertical profile of longwave cooling is regulated by the vertical profile of water vapor, which is in turn very sharp at the level of the tropopause; and (ii) PV material modified in the troposphere encounters a transport barrier at the level of the tropopause.

The scope of the present investigation was restricted to demonstrating the structure and variation of the simulated PV dipole between cases and model resolutions. The investigation has not considered the detailed mechanisms controlling the production and specific characteristics of the dipole, such as its depth, amplitude, and variation between cases. Given the role of longwave radiative cooling in its generation, the characteristics of the dipole are likely to be related to the profile of water vapor across the tropopause layer. In fact, the larger amplitude dipoles in the troughs compared to in the ridges were accompanied by larger gradients of moisture across the tropopause in the troughs (not shown); furthermore, the shallow dipole diagnosed in case II was accompanied by a sharper but shallower humidity gradient than in either of the other cases. Cavallo and Hakim $(2012,2013)$ demonstrated that longwave radiative cooling across sharp humidity gradients is responsible for the intensification of tropopause polar vortices (TPVs). Although latent heating is more abundant in extratropical cyclones, it is possible that radiative cooling plays a similar role to that in TPVs. The dipole structure may also be related to the tropopause inversion layer (TIL) in the extratropics identified by Birner et al. (2002) and Birner (2006). The TIL is a climatological feature consisting of a sharp increase in temperature above the cold point tropopause and a corresponding increase in static stability. Randel et al. (2007) proposed that the TIL is generated by a radiative forcing mechanism associated with the vertical gradients of ozone and water vapor across the tropopause. The maximum in static stability in the TIL is located in the region where the positive portion of the PV dipole forms (not shown). Randel and Wu (2010) identified a similar maximum in static stability in the polar summer tropopause inversion layer. The diabatically generated PV dipole diagnosed in this paper is likely to be controlled by similar radiative mechanisms to those that are active in both TPVs and in the TIL.

The tracer technique used in this study only permitted diagnosis of accumulated diabatic PV sources from the beginning of the model simulations; the net diabatic PV was defined as zero at the time of initialization. This property of the technique permitted an analysis of the time scales associated with diabatic PV accumulation and saturation. The dipole across the tropopause was shown to accumulate slowly and achieve a steady state after approximately $36 \mathrm{~h}$. The positive pole in the lower stratosphere, which is associated with longwave radiative cooling, accumulated more slowly than the negative pole in the troposphere, which is influenced by latent heating in the troposphere. Because the tendencies in PV remain continually active across the extratropical tropopause, the saturation in dipole amplitude must be a consequence of either vertical motion or dissipative processes counteracting the tendencies. It is not known to what extent these limiting processes and the associated 36-h saturation time are representative of real physical processes or artificial numerically dissipative processes. The semi-Lagrangian scheme that is used to advect PV tracers introduces implicit diffusion. Complicating the interpretation of the dipole saturation is the fact that mixing of PV is not as straightforward as mixing of a chemical tracer such as ozone. Haynes and McIntyre (1990) demonstrated that PV mixing by smallscale turbulence can even result in upgradient transport, such as in the case of gravity wave breaking. Nevertheless, Danielsen (1990, and references therein) have argued that PV may be treated like a chemical tracer with reasonable success to interpret the source and mixing of air in the upper troposphere and lower stratosphere. As a thought experiment, the time scale of saturation can be used to infer an eddy diffusivity for PV material near the tropopause if we assume that transport of PV by explicitly resolved vertical velocity is small across the extratropical tropopause and that PV diffuses like a chemical tracer (the limitations of which are noted above). For simplicity, we assume a local balance between PV tendencies and second-order diffusion near the tropopause:

$$
\frac{\partial q}{\partial t}+\kappa \frac{\partial^{2} q}{\partial z^{2}}=0
$$

where $q=f(z) \alpha(t)$ is the diabatic PV that has vertical structure $f(z)$ and time dependence $\alpha(t)$, and $\kappa$ is the eddy diffusivity. We assume that the vertical structure of the diabatic PV is sinusoidal:

$$
f(z)=\sin \left(\frac{\pi z}{d}\right) .
$$

Taking the maximum of the dipole to be located $1 \mathrm{~km}$ from the tropopause such that $d=1 \mathrm{~km}$ (e.g., see Fig. 2), and the rate of PV accumulation by diabatic processes, $\Delta q / \Delta t$, to be $0.5 \mathrm{PVU}$ in $24 \mathrm{~h}$, the implied eddy diffusivity is given by 


$$
\kappa=\frac{d^{2}(\Delta q / \Delta t)}{\pi^{2}|q|}=\frac{(1 \mathrm{~km})^{2} \times 0.5 \mathrm{PVU}}{\pi^{2} \times 0.5 \mathrm{PVU} \times 24 \mathrm{~h}} \approx 1 \mathrm{~m}^{2} \mathrm{~s}^{-1} .
$$

This value is consistent with the range of approximately $0.3-3 \mathrm{~m}^{2} \mathrm{~s}^{-1}$ estimated by Kurosaki et al. (1996) and Narayana Rao et al. (2001). The above calculation ignores the effect of advective transport. When averaged across the upper-level wave over several days, the mean vertical velocity in each of the three cases is small.

The dependence of diabatic PV structure on model grid resolution was shown to be insignificant, even though the highest-resolution simulations were performed without a convection parameterization scheme. Simulations performed with 1-km horizontal grid spacing (and convection parameterization disabled) did generate smaller-scale structures than simulations performed with 12- and 4-km grid spacing. However, the mean diabatic PV, averaged across the small tracer domain, was very similar in the runs. This result implies that the synoptic-scale flow exerts the primary control on the characteristics of net diabatic PV production across the tropopause in extratropical cyclones and is likely to be a consequence of (i) the dipole being the product of the gradual accumulation of PV tendencies over a broad region; (ii) the dominance of the radiative cooling, which is relatively insensitive to resolution, in the generation of the positive pole; and (iii) the net ascent in the warm conveyor belt being controlled by the largescale dynamics of the extratropical cyclone. It is not clear whether a similar result would apply in flows that demonstrate upscale organization, such as mesoscale convective systems or tropical cyclones. Done et al. (2006) showed that the characteristics of system-integrated convective available potential energy (CAPE) and precipitation as well as $\mathrm{PV}$ do depend on whether convection is parameterized or explicitly resolved, but the mesoscale convective systems analyzed were more convectively unstable than the cases presented in this paper.

Finally, the characteristics of the dipole were not uniform across the large domains in which the simulations were performed. In case I, positive net diabatic PV accumulated at the level of the tropopause (i.e., 1.55 PVU) within a subdomain confined to low latitudes south of the primary jet (not shown). This positive diabatic PV was accompanied by mean ascent of $1-3 \mathrm{~cm} \mathrm{~s}^{-1}$ across the tropopause layer. The dipole structure at these lower latitudes was distinctly different from that in extratropical latitudes. Near the primary upper-level wave, the mean ascent (not shown) in the tropopause layer was considerably less $\left(<0.5 \mathrm{~cm} \mathrm{~s}^{-1}\right)$, and the node of the dipole straddled the tropopause. The properties of diabatic PV across the extratropical tropopause that have been discussed above, and in CGM13, must, therefore, not be extended to subtropical regions until further investigation is conducted.

Acknowledgments. The lead author was supported by the National Centre for Atmospheric Science (NCAS) under the Weather Directorate. The research was also supported by the Natural Environment Research Council project "Diabatic Influences on Mesoscale Structures in Extratropical Storms" (DIAMET) as part of the Storm Risk Mitigation program (NERC Grant NE/I005196/1). John Methven and two anonymous reviewers offered valuable feedback that led to significant improvements in the discussion and interpretation of results. The authors thank Leo Saffin for identifying and calculating inconsistencies in the method of conservative PV advection. The authors also thank Willie McGinty of NCAS Computational Modelling Services (CMS) for providing data and support necessary to run the MetUM on the U.K. National Supercomputing Service, and the Met Office for making the MetUM available for research.

\section{REFERENCES}

Birner, T., 2006: Fine-scale structure of the extratropical tropopause region. J. Geophys. Res., 111, D04104, doi:10.1029/ 2005JD006301.

- A. Dornbrack, and U. Schumann, 2002: How sharp is the tropopause at midlatitudes? Geophys. Res. Lett., 29, 1700, doi:10.1029/2002GL015142.

Brennan, M. J., G. M. Lackmann, and K. M. Mahoney, 2008: Potential vorticity (PV) thinking in operations: The utility of nonconservation. Wea. Forecasting, 23, 168-182, doi:10.1175/ 2007WAF2006044.1.

Cavallo, S. M., and G. J. Hakim, 2012: Radiative impact on tropopause polar vortices over the Arctic. Mon. Wea. Rev., 140, 1683-1702, doi:10.1175/MWR-D-11-00182.1.

— and - 2013: The physical mechanisms of tropopause polar cyclone intensity change. J. Atmos. Sci., 70, 3359-3373, doi:10.1175/JAS-D-13-088.1.

Chagnon, J. M., S. L. Gray, and J. Methven, 2013: Diabatic processes modifying potential vorticity in a North Atlantic cyclone. Quart. J. Roy. Meteor. Soc., 139, 1270-1282, doi:10.1002/qj.2037.

Danielsen, E. F., 1990: In defense of Ertel's potential vorticity and its general applicability as a meteorological tracer. J. Atmos. Sci., 47, 2013-2020, doi:10.1175/1520-0469(1990)047<2013: IDOEPV $>2.0 . \mathrm{CO} ; 2$.

Davies, H. C., and M. Didone, 2013: Diagnosis and dynamics of forecast error growth. Mon. Wea. Rev., 141, 2483-2501, doi:10.1175/MWR-D-12-00242.1.

Davies, T., M. J. P. Cullen, A. J. Malcolm, M. H. Mawson, A. Staniforth, A. A. White, and N. Wood, 2005: A new dynamical core for the Met Office's global and regional modelling of the atmosphere. Quart. J. Roy. Meteor. Soc., 131, 1759-1782, doi:10.1256/qj.04.101.

De Vries, H., J. Methven, T. H. A. Frame, and B. J. Hoskins, 2010: Baroclinic waves with parameterized effects of moisture interpreted using Rossby wave components. J. Atmos. Sci., 67, 2766-2784, doi:10.1175/2010JAS3410.1. 
Done, J. M., G. C. Craig, S. L. Gray, P. A. Clark, and M. E. B. Gray, 2006: Mesoscale simulations of organized convection: Importance of convective equilibrium. Quart. J. Roy. Meteor. Soc., 132, 737-756, doi:10.1256/qj.04.84.

Edwards, J. M., and A. Slingo, 1996: Studies with a flexible new radiation code: I. Choosing a configuration for a large scale model. Quart. J. Roy. Meteor. Soc., 122, 689-720, doi:10.1002/ qj.49712253107.

Gregory, D., and P. R. Rowntree, 1990: A mass flux convection scheme with representation of cloud ensemble characteristics and stability-dependent closure. Mon. Wea. Rev., 118, 1483-1506, doi:10.1175/1520-0493(1990)118<1483:AMFCSW>2.0.CO;2.

Harrold, T. W., 1973: Mechanisms influencing the distribution of precipitation within baroclinic disturbances. Quart. J. Roy. Meteor. Soc., 99, 232-251, doi:10.1002/qj.49709942003.

Haynes, P. H., and M. E. McIntyre, 1990: On the conservation and impermeability theorems for potential vorticity. J. Atmos. Sci., 47, 2021-2031, doi:10.1175/1520-0469(1990)047<2021: OTCAIT $>2.0 . \mathrm{CO} ; 2$.

Heifetz, E., C. H. Bishop, B. J. Hoskins, and J. Methven, 2004: The counter-propagating Rossby-wave perspective on baroclinic instability. I: Mathematical basis. Quart. J. Roy. Meteor. Soc., 130, 211-231, doi:10.1256/qj.02.184.

Hoerling, M. P., T. K. Schaack, and A. J. Lenzen, 1991: Global objective tropopause analysis. Mon. Wea. Rev., 119, 1816-1831, doi:10.1175/1520-0493(1991)119<1816:GOTA > 2.0.CO;2.

Kunz, A., P. Konopka, R. Müller, and L. L. Pan, 2011: Dynamical tropopause based on isentropic potential vorticity gradients. J. Geophys. Res., 116, D01110, doi:10.1029/2010JD014343.

Kuo, Y. H., M. A. Shapiro, and E. G. Donall, 1991: The interaction between baroclinic and diabatic processes in a numerical simulation of a rapidly intensifying extratropical marine cyclone. Mon. Wea. Rev., 119, 368-384, doi:10.1175/1520-0493(1991)119<0368: TIBBAD $>2.0 . \mathrm{CO} ; 2$.

Kurosaki, S., M. Yamamoto, H. Hashiguchi, T. Sato, and S. Fukao, 1996: Vertical eddy diffusivity in the lower and middle atmosphere: A climatology based on the MU radar observations during 1986-1992. J. Atmos. Sol.-Terr. Phys., 58, 727-734, doi:10.1016/0021-9169(95)00070-4.

Lock, A. P., A. R. Brown, M. R. Bush, G. M. Martin, and R. N. B. Smith, 2000: A new boundary layer mixing scheme. Part I: Scheme description and single-column model tests. Mon. Wea. Rev., 128, 3187-3199, doi:10.1175/1520-0493(2000)128<3187: ANBLMS $>2.0 . \mathrm{CO} ; 2$
Martinez-Alvarado, O., H. Joos, J. M. Chagnon, M. Boettcher, S. L. Gray, R. S. Plant, J. Methven, and H. Wernli, 2014: The dichotomous structure of the warm conveyor belt. Quart. J. Roy. Meteor. Soc., 140, 1809-1824, doi:10.1002/qj.2276.

Moore, R. W., and M. T. Montgomery, 2004: Reexamining the dynamics of short-scale, diabatic Rossby waves and their role in midlatitude moist cyclogenesis. J. Atmos. Sci., 61, 754-768, doi:10.1175/1520-0469(2004)061<0754: RTDOSD $>2.0 . \mathrm{CO} ; 2$.

— - _ and H. Davies, 2013: Genesis criteria for diabatic Rossby vortices: A model study. Mon. Wea. Rev., 141, 252263, doi:10.1175/MWR-D-12-00080.1.

Narayana Rao, D. N., M. V. Ratnam, T. N. Rao, and S. V. B. Rao, 2001: Seasonal variation of vertical eddy diffusivity in the troposphere, lower stratosphere and mesosphere over a tropical station. Ann. Geophys., 19, 975-984, doi:10.5194/ angeo-19-975-2001.

Parker, D. J., and A. J. Thorpe, 1995: Conditional convective heating in a baroclinic atmosphere: A model of convective frontogenesis. J. Atmos. Sci., 52, 1699-1711, doi:10.1175/ 1520-0469(1995)052<1699:CCHIAB > 2.0.CO;2.

Randel, W. J., and F. Wu, 2010: The polar summer tropopause inversion layer. J. Atmos. Sci., 67, 2572-2581, doi:10.1175/ 2010JAS3430.1.

— W. Fei, and P. Forster, 2007: The extratropical tropopause inversion layer: Global observations with GPS data, and a radiative forcing mechanism. J. Atmos. Sci., 64, 4489-4496, doi:10.1175/2007JAS2412.1.

Roberts, N. M., 2003: The impact of a change to the use of the convection scheme to high resolution simulations of convective events. Met Office Forecasting Research Tech. Rep. 407, Met Office, $31 \mathrm{pp}$.

Stoelinga, M. T., 1996: A potential vorticity-based study of the role of diabatic heating and friction in a numerically simulated baroclinic cyclone. Mon. Wea. Rev., 124, 849-874, doi:10.1175/ 1520-0493(1996)124<0849:APVBSO>2.0.CO;2.

Wernli, H., and H. C. Davies, 1997: A Lagrangian-based analysis of extratropical cyclones. I: The method and some applications. Quart. J. Roy. Meteor. Soc., 123, 467-489, doi:10.1002/ qj. 49712353811.

Wilson, D. R., and S. P. Ballard, 1999: A microphysically based precipitation scheme for the UK Meteorological Office Unified Model. Quart. J. Roy. Meteor. Soc., 125, 1607-1636, doi:10.1002/qj.49712555707. 